\title{
Aportes de la paleopatología en la interpretación arqueológica
}

\author{
Contributions in the archaeological interpretation Paleopathology
}

\author{
Denis Correa Trigoso ${ }^{1}$
}

\section{RESUMEN}

La investigación arqueológica está enfocada en la exposición de diversos resultados obtenidos de los análisis paleopatológicos de los restos óseos provenientes de los contextos arqueológicos en los Andes Centrales. Para esta finalidad se utilizó los estudios más relevantes en antropología física publicados hasta la actualidad asociada con el área andina; así como conceptos básicos para lograr comprender correctamente los procesos que existieron para la formación de patologías en los restos óseos. De esta manera se logra determinar que ciertos tipos de patologías presentes en los Andes Centrales puedan estar asociadas a cambios económicos, inmolaciones rituales, modificaciones corporales, intervenciones médicas e implicancias medioambientales.

Palabras clave: paleopatología; arqueología; óseo; antropología física.

\begin{abstract}
The research is focused on the exposure of the various results of present paleopathological analysis in the skeletal remains from archaeological contexts in the central Andes. The most relevant studies was used in physical anthropology published to date associated with the Andean region for this purpose. Additional basic concepts to correctly understand the processes that existed for the formation of bone diseases. Thus it was determined that certain types of lesions present in the central Andes can be associated with economic, ritual immolations, body modification, medical interventions and environmental implications.
\end{abstract}

Keywords: paleopathology; archeology; skeletal; physical anthropology.

\section{ICHIKLLACHAW}

Kay musyapakuychawqa willakun tukuy laaya uryatam, kaychaw nin imanaw tukuy laaya risultadukuna palyupatuluhikukunapa analisis tullukunapa kanqanta, tsay arkiyuluhiyakuna imanawmi andispa chawpinchaw kanqanta.

Kay llapan allin kaq yachakuykunata rurakashqa antrupuluhiya huwisika nishqanta kanankama riqitsikushqanta, kayqa andispa patsanchaw tinkushqantam, tsaynawpa shumaq qillqaqkuna alliq kaayinapaq hina llapan prusisukunata kawanqanta kaayirinapaq, patuluhiyakuna tulluchaw nishqan rurashqantam. Tsaynawpam alli yarqunaq wakin patuluhiyakunapa laayankuna andiskunapa chawpinchaw, kaykuna kanmanmi: ikunumikukuna tikrayninchaw, inmulasiyunkunachaw, ritwalkunachaw, kurpuralis nishqan huk laaya tikrayninkunachawwan hina midikakunawan midyu ambiyintalkuna nishqanchawwan.

1 Universidad Nacional de Trujillo. La Libertad, Perú. 
Pushaq shimikuna: palyupatuluhiya; arqiyuluhiya; tullu; antrupuluhiya huwisika nishqan.

\section{INTRODUCCIÓN}

El ser humano se ha establecido en diversos tipos de territorios, lo cual conllevó a afrontar las diversas dificultades que el entorno le imponía, siendo un caso más directo a nuestra realidad las numerosas sociedades que se establecieron en el Perú prehispánico. La adaptación del poblador prehispánico y la relación con su entorno se encuentran evidenciadas en el registro arqueológico. Las sociedades andinas lograron desarrollarse a pesar de las complicaciones que presentaba el lugar donde se establecieron y prosperaron al modificar su entorno a su beneficio, cuyas evidencias se ven en las construcciones de múltiples obras de irrigación y de plataformas de cultivos en las faldas de los cerros. La adaptación a este nuevo entorno tanto social como biológico generó alteraciones que estuvieron relacionadas directamente con el modo de vida de cada individuo. A este respecto formulamos dos preguntas: ¿Cómo se refleja la adaptación en el individuo? y ¿De qué manera analizar estas adaptaciones puede aportar a la interpretación arqueológica? Para lograr responder estas interrogantes se abordará el campo de la Antropología Física, el cual nos brindará pistas aproximadas y específicas sobre las respuestas osteológicas que surgieron a partir de los diversos procesos adaptativos (cualquiera que sea su naturaleza), teniendo una gran importancia para este fin las alteraciones óseas que los restos puedan exhibir.

La presente investigación tiene como objetivo conocer cuáles son los aportes que genera el análisis paleopatológico en la interpretación arqueológica, a partir del reconocimiento de las alteraciones registradas en los restos óseos y las representaciones en el material cerámico procedente del contexto arqueológico.

\section{METODOLOGÍA}

La presente investigación es una revisión de los resultados obtenidos de los análisis bioantropológicos realizados en los Andes Centrales. Para el marco teórico se ha utilizado múltiples publicaciones científicas relacionadas con la Antropología Física, con la finalidad de lograr obtener los conceptos y definiciones que ayuden con el desarrollo de la investigación. Asimismo se ha empleado los resultados obtenidos de los estudios paleopatológicos en el material arqueológico, para poder establecer cuáles fueron sus implicancias en la interpretación arqueológica.

\section{RESULTADOS Y DISCUSIÓN}

\section{La paleopatología}

El ser humano en la actualidad sufre de diversas enfermedades (patologías) ocasionadas por un sinnúmero de causas de las cuales la mayoría pueden ser detectadas, pero si a este enunciado se le cambia de contexto y se traslada hace miles de años atrás ¿Cómo detectaríamos estas enfermedades? y ¿De qué manera reconoceríamos ciertos males sufridos por el ser humano? La disciplina que estudiaría estos padecimientos en el contexto arqueológico sería la paleopatología, cuyo nombre deriva de los vocablos griegos: paleo (viejo) y patos (sufrimiento). Es definido como el estudio de 
las enfermedades de pueblos antiguos, teniendo como fuentes el estudio de los restos humanos (momias, huesos, dientes, coprolitos), las informaciones históricas, y la representación de las enfermedades en las artes pictóricas o escultóricas (Sotomayor y Cuéllar-Montoya, 2007: 5).

Un punto importante es lograr determinar la naturaleza de las alteraciones en los huesos y el momento exacto durante el cual se produjeron; en caso contrario conllevarían a generar resultados erróneos y por ende una comprensión equivocada sobre el origen de las alteraciones. Se puede definir en 3 grupos muy distintos (Etxeberria et al., 2005: 346):

- Antemortem: Relacionada de forma directa o indirecta con el resultado final o fallecimiento del sujeto.

- Perimortem: Con relación directa, o sin ella, al resultado final de la muerte.

- Postmortem: Sin relación alguna con el fallecimiento y asociada con la manipulación de los restos, como por ejemplo algún tipo de ritual funerario (enterramiento primario o secundario).

Se debe tener en consideración los factores tafonómicos (tipo de enterramiento, insectos, intemperie, agua, etc.) que han intervenido sobre los restos óseos que podrían interferir en su estudio y llegar a ser confundidos con patologías (comunicación personal Amália Valls, 2012). Es así que al conocer todos estos aspectos podemos determinar de una forma más precisa cuáles serían las enfermedades y/o alteraciones artificiales que padecieron las antiguas poblaciones en los Andes Centrales.

\section{La paleopatología en los Andes Centrales}

Producto de los análisis realizados al material óseo fue posible lograr tener mayores aproximaciones a los cambios económicos, inmolaciones rituales, modificaciones corporales, intervenciones médicas e implicancias medioambientales a que estuvieron expuestos los diversos grupos asentados en los Andes Centrales.

\subsection{Cambios económicos}

La supervivencia de los individuos se encuentra relacionada con las distintas estrategias usadas por los grupos para afrontar diversos problemas, sean de carácter social o ambiental, y asegurarse la permanencia en un área determinada. Una manera de hacer frente a estas dificultades es el uso de una economía determinada, como la ejercida por los asentamientos precerámicos presentes en la costa, los cuales tuvieron al mar como proveedor de recursos, originando de esta forma la práctica de la pesca y la recolección de mariscos. Sin embargo, un cambio económico representa variaciones en las técnicas de explotación de recursos, el cual generaría estrés en la población y que se vería reflejado en el cuerpo, particularmente en algunos tipos de patologías.

Estos cambios se encuentran evidenciados en el sitio de Puémape, una población pesquera del formativo (2500-1 a.C). Se logró determinar que durante los distintos periodos de ocupación hubo cambios en los modos de subsistencia y en el rol social que ejercían estas actividades. Durante el periodo temprano se tiene la mayor presencia de exostosis auditiva (unilateral o bilateral), un indicador óseo relacionado con actividad 
acuática (buceo, pesca, nado). En periodo medio la actividad acuática disminuye y es acompañada con una mayor incidencia de exostosis auditiva en mujeres, sugiriendo un posible cambio de roles y una mayor participación femenina en el trabajo de extracción dentro de una nueva distribución de funciones. Es en la época tardía que la presencia de esta patología es escasa y focalizada, lo cual se relaciona con cambios sociales que influyeron en el modo de subsistencia (la agricultura toma un rol más importante en esta población) y se asocia estos pocos casos con la especialización de cierto sector de los habitantes con labores relacionadas a la extracción marina (Pezo-Lanfranco et al., 2009).

\subsection{Inmolaciones rituales}

La explicación de los fenómenos naturales presentes en el entorno estuvieron asociadas según la presión social e ideológica dentro del grupo, lo que genera una reacción para demostrar devoción y su relación con la deidad tutelar. Una respuesta a estos ideales se puede encontrar en la inmolación o sacrificio humano, puesto que este acto moral demuestra el desprendimiento y privación de algo de valor a fin de obtener algún beneficio como resultado final (Gonzales, 2006).

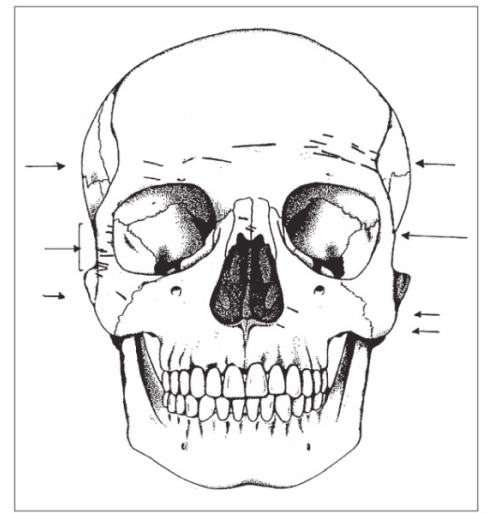

Figura 1. Cráneo con cortes en el rostro asociados con el descarnamiento

(Verano et al., 2007)

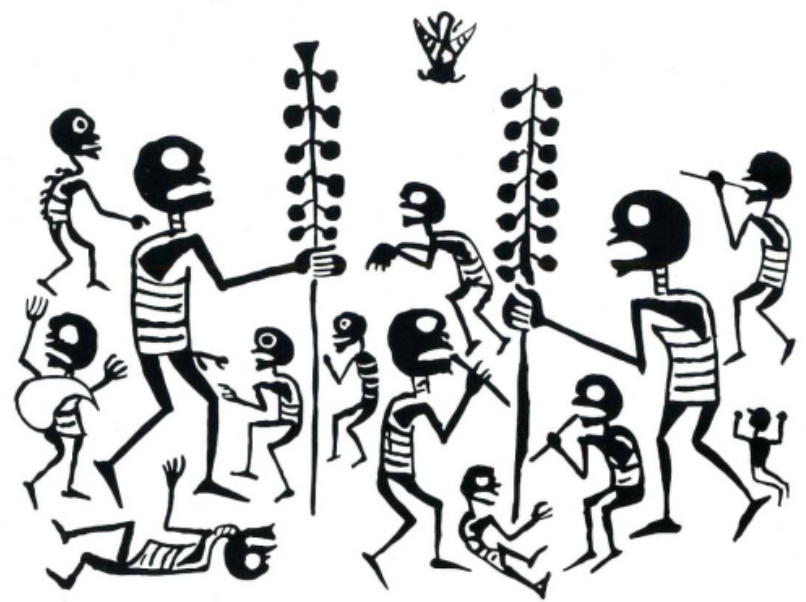

Figura 2. Escena de figuras esqueléticas con instrumentos musicales, posiblemente en un acto ritual (Hocquenghem, 1989:337) 
Un caso muy investigado es el análisis óseo de los restos mochica (Verano, 1994, 1997, 1998, 2003; Backo, 2009), los cuales demuestran lesiones de cortes sobre la cara anterior de los cuerpos vertebrarles y también en las apófisis traversas (entre la C3 y C5), esto manifiesta actos de degollamiento que encontramos reflejados en la iconografía y cerámica (Donnan, 1978); además se evidencia decapitaciones y traumas de naturaleza interpersonal (Verano, 2008). Estos restos se asocian con sacrificios (precaptura) y combates rituales celebrados dentro de un marco ritual para conmemorar actividades religiosas, las cuales guardaban un propósito más definido que era la captura de individuos para el sacrificio a sus divinidades y el uso de su sangre como bebida por sus dioses-hombres (posiblemente los gobernantes) que dirigían las ceremonias (Castillo, 2000).

Existen múltiples planteamientos asociados con las razones que motivaron a los mochicas a desmembrar y a descarnar a sus sacrificados. Verano et al. (2007) propone que aunque se pueden asociar con actividades de antropofagia aún no es posible asegurar tal acto. Para Carod-Artal y Vázquez-Cabrera (2007), los cuerpos eran descarnados sin comprometer los tendones para su posterior utilización en danzas rituales, donde los danzarines portaban sobre sus hombros esos esqueletos articulados y luego debido a la descomposición de la materia blanda terminaban desarticulándose (figura 1 y 2).

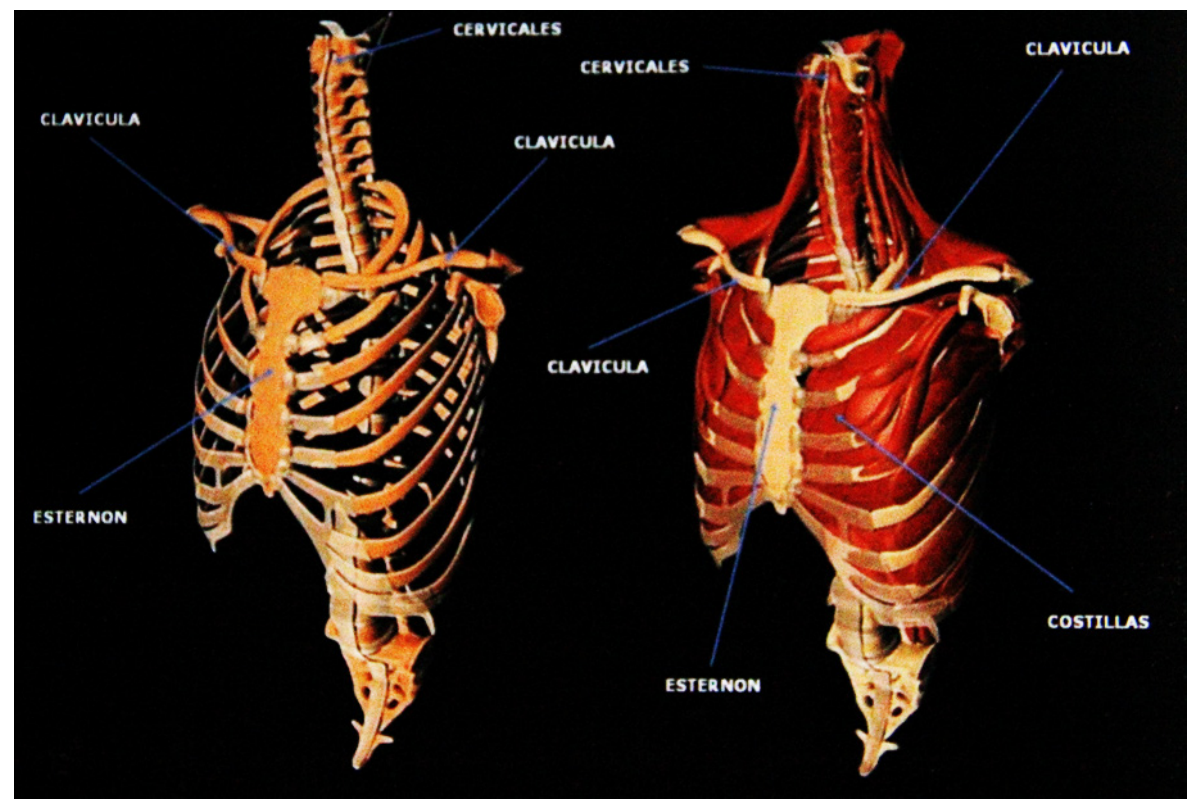

Figura 3. Localización de los cortes identificados para los sacrificios Lambayeque en la Huaca Los Sacrificios, vinculados con la abertura del pecho con la finalidad de llegar al corazón (Wester 2010)

Una manera distinta de realizar el mismo acto se ve reflejada en los sacrificios efectuados por la cultura Lambayeque. Wester (2010: 94-104), registra que a pesar de realizar degollamientos se tuvo una manera distinta de hacer estos actos y esto estuvo vinculado con las variadas formas de aberturas del pecho (cortes en la clavícula y costillas). Estas fracturas son consecuencia de la abertura fuerte y violenta de la cavidad pectoral hecha con la mano para obtener el corazón de la víctima (figura 3). Todos estos sacrificios se asocian con la intención de extraer la mayor cantidad de sangre y otros órganos. 


\subsection{Modificaciones corporales}

Las culturas demuestran su particularidad a través de ciertas manifestaciones, las cuales son excluyentes pero a su vez unificadoras; un claro ejemplo son las prácticas de la modificación cultural de la cabeza, presentes en distintos periodos y en variadas culturas (figura 4). Existen cuatro diferentes tipos de modificaciones craneales (Weiss, 1961; Tello y Mejía, 1979; Dembo e Imbelloni, 1938; Yépez, 2006):

- Modelado cefálico anular: Comprimido el cráneo circularmente tomando forma de un cilindro: circular (recto)-recto, con su subtipo de oblicuo y erecto de formas esferocilíndricas.

- Tabular cilíndrico o tabular oblicuo: Presentan hendidura pronunciada en la escama media del frontal.

- Cuneiforme, Tabular erecta o Tipo Cavernas: Con el occipital aplanado y frontal alargado oblicuamente.

- Fronto-Occipital Erecto, variedad bilobulada: Se tiene el frontal y occipital erectos con los parietales ensanchados.

Según Weiss (1961) cada tipo de deformación se relaciona con un apero en particular: por llautu (únicamente bandas y cintas) y con roscas o almohadillas de algodón. También existen deformaciones causadas por cuna, la cual se caracteriza por su apariencia asimétrica, irregular y se diferencia por el aplanamiento en la zona frontal (fronto-occipital), puesto que los niños eran amarrados en sus cunas desde temprana edad propiciando de tal forma su deformación (figura 5).

A través del tiempo es posible relacionar un tipo de deformación craneal con una sociedad determinada, como las presentes en la cultura material de las poblaciones Nazca, Chimú, Paracas, Recuay, Mochica y Chancay (figura 6), no siendo esta la única alteración practicada, sino la más recurrente en el registro arqueológico, asimismo es posible rastrear la presencia de diversas formas durante algunos periodos de tiempo (Yépez, 2006):

- Se tiene primeras evidencias de deformaciones artificiales para el hombre de Lauricocha (Etapa lítica) asociadas al tipo tabular erecto.

- Durante el periodo precerámico en sitios como Culebras y Asia, las deformaciones corresponden a los tipos tabular erecto, de cuna rígida y vendaje frontal; además del tipo fronto-occipital erecto.

- En el Horizonte Temprano o Formativo (1800 años a.C. y 100 d.C.) y el Periodo Intermedio Temprano (100 años d.C. a 700 años d.C.) se tiene la forma tabular oblicua o llamados cabeza alargada.

- En el Periodo Intermedio Tardío se tiene con frecuencia a los tipos fronto-occipital erectos y cuneiformes, pero se observa un decaimiento en el modelar de cabezas ya que hay un mayor porcentaje de cráneos no modelados.

- Durante el Horizonte Tardío (1400 d.C. a 1532 d.C.) las prácticas deformadoras fueron decayendo y las formas fueron regionales ya que el Inca permitía mantener sus usos y costumbres. 

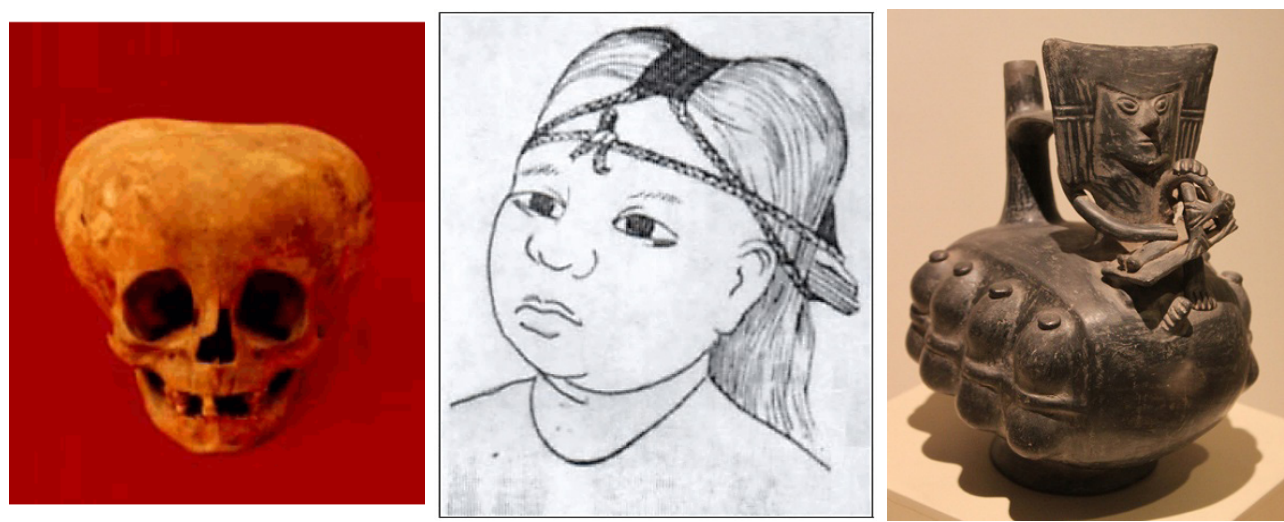

Figuras 4, 5 y 6. Cráneo Chancay de un infante de 6 a 8 años con deformación de forma bilobular del tipo fronto-occipital oblicuo (izquierda) y el posible apero modelador utilizado para realizar este tipo de deformación (centro) (Yépez y Arzápalo, 2007), además cerámica

Chimú con la misma deformación presente en grupos Chancay (MNAAHP) (derecha).

Se debe tener en cuenta la naturaleza primigenia del motivo de esta práctica, no es simplemente el deseo de cambiar la forma del cráneo o de obedecer criterios estéticos, sino de ver al cuerpo humano como una vía de comunicación (texto), donde el emisor va a producir un mensaje que está conformado por signos y códigos, los cuales generan una respuesta social causada por la interpretación del receptor a partir de su experiencia acumulada dentro de un contexto cultural determinado (Yépez, 2006). Estos mensajes tenían como finalidad generar la diferenciación notoria del grupo que lo practicaba puesto que hubo sectores de la población que no lo practicaban; además este comportamiento servía para distinguir épocas y relaciones, posibles categorías sociales y raíces culturales. El cronista De Las Casas (1909) menciona que los señores incas y su estirpe practicaron tres diferentes tipos de deformación de cabeza y que de estos actos les fue avisado a los distintos señores y linajes dentro del territorio para que tengan conocimiento de su significado; asimismo se hace referencia que era una práctica impuesta por el Inca y obligaba a la población a deformarse la cabeza de una manera específica (cabezas largas con la frente quebrada) porque esto las hacía simples y sin ánimos, caso contrario el de los no deformados (gran cabeza y redonda) que tenían un carácter inobediente.

\subsection{Intervenciones médicas}

Las amputaciones quirúrgicas se demuestran en las evidencias óseas, donde los extremos distales de ambas tibias y peroné muestran proliferación ósea extensa y superficies articulares afuncionales, sugiriendo amputaciones curadas (Verano y Lombardi, 1999). Estas prácticas están presentes en la cerámica, donde se plasman individuos sin brazos, antebrazos, manos o pies. Es muy clara la presencia de una depresión o surco muy visible al medio de cada muñón de las piernas relacionada con la desarticulación desde el tobillo y el uso de copas sobre la base terminal a manera de prótesis para poder caminar luego de la intervención (figura 7), (Verano, 2003). 

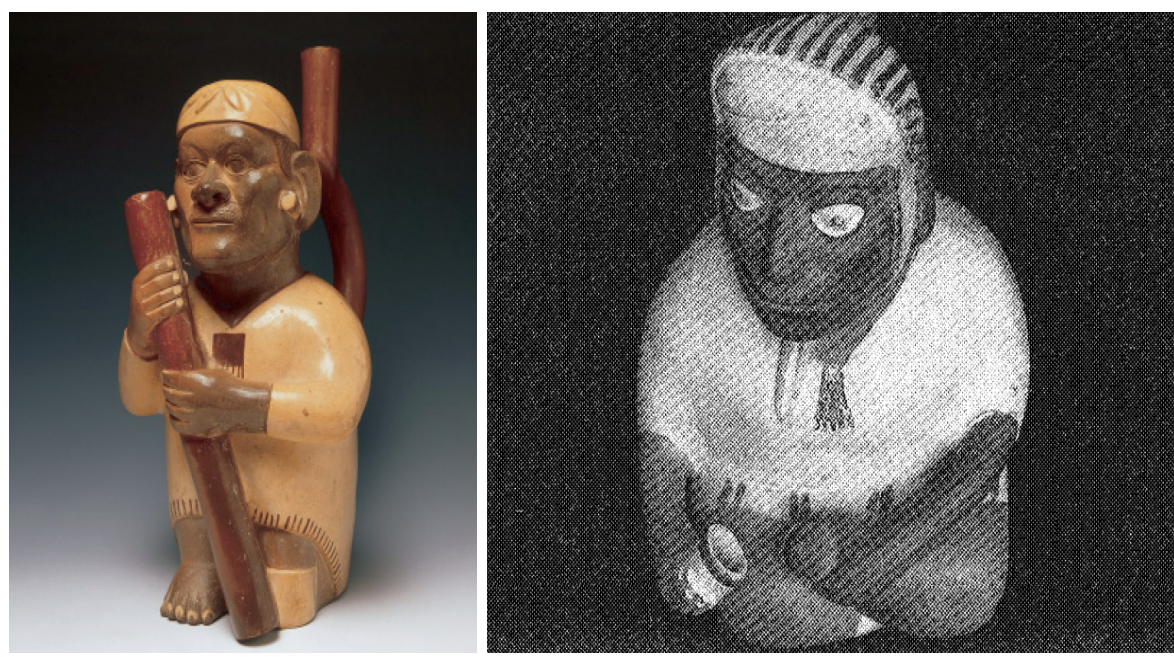

Figura 7. Un individuo que demuestra la amputación del pie izquierdo con utilización de prótesis (Larco, 2001) (izquierda) y sujetando el pie para colocar una prótesis

(derecha) (Verano y Lombardi, 1999).

Para Idrovo (2005) estas prácticas quirúrgicas en los pies estaban asociadas con el padecimiento llamado «enfermedad del pie negro» (Cylindrocarpon spp.) y el de las extremidades por la intoxicación aguda relacionada con la exposición excesiva al arsénico.

\subsection{Implicancias ambientales}

El medio ambiente no es simplemente una fuente de recursos para su explotación, sea de materia prima o de alimentos, sino también juega un papel influyente en la elección sobre el tipo de organización y actividades de supervivencia (estrategias) utilizadas por un grupo en particular (Alfaro, 2006). Por esta razón cualquier actividad que realicen los integrantes de estos grupos se verá reflejada en las consecuencias que conllevaran para sus vidas, y en caso más específico los que implican presencia de variaciones en la morfología ósea. El registro de hipoplasia del esmalte (figura 8), criba orbitaria, hiperostosis porótica (figura 9) se relaciona con deficiencias en la dieta, lo cual se puede asociar con grupos de estatus socioeconómicos bajos, ya que al no tener acceso a diferentes recursos para constituir una dieta con mayor valor nutricional solo se consumió alimentos disponibles localmente, ejemplo de esto son los individuos sacrificados que fueron analizados en la Huaca de Los Sacrificios, en el Complejo Arqueológico Chotuna-Chornacap (Wester, 2010).
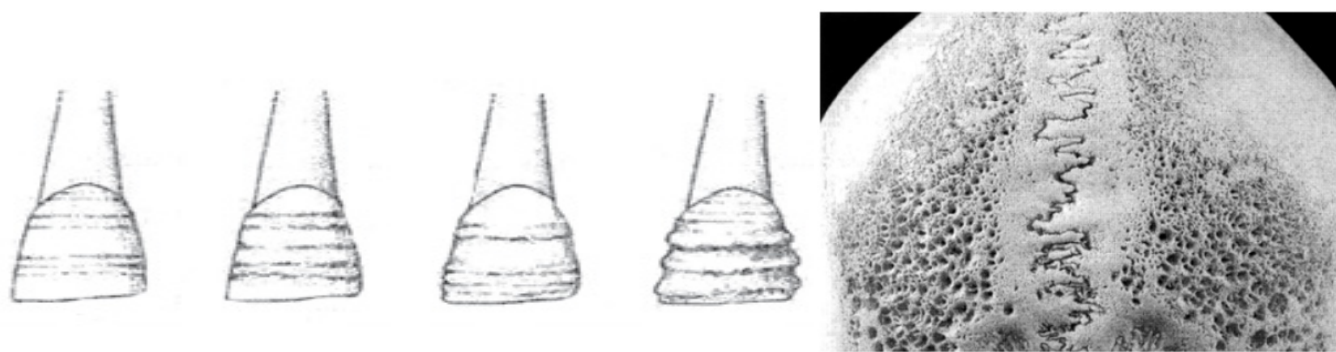

Figuras 8 y 9. Formas de hipoplasia de esmalte en el incisivo superior (izquierda) y la presencia marcada de hiperostosis porótica en un cráneo (derecha) (Krenzer, 2006) 
De otra manera, tener acceso constante al agua dulce es una de las principales necesidades a suplir para la supervivencia de un grupo humano y estas fuentes hídricas a su vez tienen una relación directa con su entorno geoclimático, por tal motivo su consumo puede producir ciertas afecciones particulares que pueden ser reconocibles, como es el caso de la fluorosis dental, esta anomalía de la cavidad oral es originada por la ingesta excesiva y prolongada de flúor que genera un defecto en la formación del esmalte dental, dando un aspecto poroso (Gómez et al., 2002) o veteado. Cada zona geográfica presenta colores característicos que se relacionan con las particularidades que presenta el entorno ambiental (marrón terroso oscuro o blanco pardo) (Valdivia, 1988). Existen zonas fluoradas en ciertas áreas costeras, ubicadas apartadas de los ríos, donde la fuente principal era la napa freática, como es el caso de Ancón, Paracas, Chongos, Wacapuy. Estas sociedades consumían agua extraída de pozos según lo corrobora el registro arqueológico, siendo este indicador paleopatológico importante para la asociación con el lugar de procedencia de un grupo o un individuo determinado.

\section{CONCLUSIONES}

Los análisis paleopatológicos de los restos óseos presentan un aporte importante a la interpretación arqueológica, puesto que contribuyen a obtener un panorama distinto para la explicación de las particularidades del contexto social y natural que tuvieron los individuos durante la vida y la muerte.

Para obtener una adecuada interpretación de las paleopatologías presentes en el material óseo es muy importante lograr establecer cual fue la naturaleza de aquellas alteraciones, ya que pueden generarse interpretaciones erróneas si no se consideran tanto el ciclo de vida del individuo como los procesos taxonómicos que pueden haber sufrido los restos.

\section{AGRADECIMIENTOS}

Deseo expresar mis más profundos agradecimientos a los distintos profesionales que me apoyaron en la elaboración de la presente investigación, que con sus críticas, recomendaciones y su paciencia me ayudaron a mejorar tanto en lo académico como en lo personal. Muchas gracias a: Dra. Rosaura Yépez Vásquez (Universidad Nacional Autónoma de México), Mercedes González y Amalia Valls (Instituto de Estudios Científicos en Momias); y a mis colegas Zayra Mendoza Eustaquio y Eduardo Eche Vega.

\section{REFERENCIAS BIBLIOGRÁFICAS}

Alfaro, Martha. 2008. «Hombre vs. Naturaleza. Adaptación biocultural de los grupos prehispánicos de cazadores-recolectores-pescadores del Golfo de Baja California Sur». En Tendencias actuales de la bioarqueología en México. ENAH-INAH-Conacultal Promep. México. 
Backo, Heather. 2009. «Anexo 1, Análisis de restos óseos - Análisis osteológico de los restos humanos sacrificatorios de la plataforma III, Huaca de la Luna, Valle de Moche, Perú». En Proyecto Arqueológico Huaca de la Luna. Trujillo: Facultad de Ciencias Sociales de la Universidad Nacional de Trujillo. 173-180.

Castillo, Luis. 2000. «La gesta del guerrero». En Mundo peruano antiguo: una visión interdisciplinaria. Lima: Pontificia Universidad Católica del Perú. 5-10.

De las Casas, Bartolomé. 1909. Apologética historia de las Indias. Por M. Serrano y Sanz. Madrid: Bailly, Bailliere e hijos.

Dembo, Adolfo y Imbelloni, José. 1938. Deformaciones intencionales del cuerpo bumano de carácter étnico. Buenos Aires: Anesi.

Donnan, Christopher. 1978. Moche art of Perú:Pre-Columbian Symbolic Communication. 1 ra. ed. Los Angeles: Museum of Cultural History, University of California.

Etxeberria, Francisco; Herrasti, Lourdes y Bandres, Antxon. 2005. «Muertes violentas determinadas a través de los estudios de paleopatología». En Munibe AntropologíaArqueología. No $57.345-357$.

Gómez, Gladys; Gómez, Dulce y Martin, Macrina. 2002. Flúor y fluorosis dental. Pautas para el consumo de dentifricos y aguas de bebida en Canarias. 1 ra. ed. Santa Cruz de Tenerife: Dirección General de Salud Pública. Servicio Canario de la Salud.

Gonzales, Yolotl. 2006. El sacrificio Humano entre los Mexicas. 1ra. reimpresión. México D.F.: Conaculta-INAH.

Hocquenghem, Anne. 1989. Iconografía mochica. 3ra. ed. Lima: Pontificia Universidad Católica del Perú.

Idrovo, Alvaro. 2005. «Posibles efectos en la salud asociados con la metalurgia precolombina». Biomédica. $\mathrm{N}^{\circ}$ 25. 295-303.

Krenzer, Udo. 2006. Compendio de métodos antropológicos forenses para la reconstrucción del perfil osteo-biologico. Guatemala: Centro de Análisis Forenses y Ciencias Aplicadas.

Larco, Rafael. 2001. Los Mochicas. Tomo 2. La Crónica y Variedades S.A.

Pezo-Lanfranco, Luis; Pezo-Lanfranco, Sandro y Eggers, Sabine. 2009. «Exostosis auditiva como marcador osteológico de actividad acuática en poblaciones formativas de la costa norte del Perú». Paleopatología. Vol. 6.

Sotomayor Hugo y Cuéllar-Montoya Zoilo. 2007. Aproximaciones a la paleopatología en América Latina. Bogotá: Convenio Andrés Bello. 
Tello, Julio y Mejía, Toribio. 1979. Paracas Cavernas y Necropolis. Vol. 2. Lima: Institute of Andean Research (New York), Universidad Nacional Mayor de San Marcos.

Valdivia, Luis. 1988. Odonto-antropología Peruana. Lima: Consejo Nacional de Ciencia y Tecnología.

Verano, John. 1994. «Características físicas y biología osteológica de los Moches». Investigaciones en la Huaca de la Luna. Trujillo: Universidad Nacional de La Libertad. 159-171.

Verano, John. 1997. «Physical Characteristics and skeletal biology of the Moche population at Pacatnamu». The Pacatnamu Papers. Vol. 2. Los Angeles: Flower Museum of Cultural History. 189-214.

Verano, John. 1998. «Sacrificios humanos, desmembramientos y modificaciones culturales en restos osteológicos: evidencias de las temporadas de investigación 1995-1996 en la Huaca de la Luna». Investigaciones en la Huaca de la Luna. Trujillo: Universidad Nacional de La Libertad. 159-171.

Verano, John. 2003. «Avances en la bioantropología de los Moche». Moche: Hacia el final del milenio. Tomo II. Trujillo: Universidad Nacional de La Libertad y Fondo Editorial de la Pontificia Universidad Católica del Perú. 15-32.

Verano, John. 2008. «Communality and Diversity in Moche Human Sacrifice». The Art and Archaeology of the Moche: An Ancient Andean Society of the Peruvian North Coast. Austin: University of Texas Press. 195-213.

Verano, John y Lombardi, Guido. 1999. «Paleopatología en Sudamérica Andina». Bulletin de l'IFEA. Tomo 28, N¹.91-121.

Verano, John, Tufinio, Moises y Lund Valle, Mellisa. 2007. «Esqueletos humanos de la plaza 3c de Huaca de la Luna». Investigaciones en la Huaca de la Luna 2001. Trujillo: Universidad Nacional de La Libertad. 225-245.

Weiss, Pedro 1961. Osteología cultural. Practicas cefálicas. Tipología de las formas cefálicas- estudio cultural de los tipos cefálicos y de algunas enfermedades óseas. Segunda parte. Lima.

Wester, Carlos. 2010. Templos, Rituales y Ancestros Lambayeque. Lambayeque: Museo Arqueológico Nacional Bruning.

Yépez, Rosaura. 2006. La práctica de modelar la cabeza en dos culturas andinas del Antiguo Perú: Paracas y Chancay. Un estudio de los procesos de significación de la cabeza modelada intencionalmente. Tesis Doctoral. México D.F.: Universidad Nacional Autónoma de México. 
| Denis Correa

Fecha de recepción: 27 de febrero de 2015

Fecha de aceptación: 04 de junio de 2015

\section{Correspondencia}

Denis Correa Trigoso

denis_1627@hotmail.com 